Santa Clara University

Scholar Commons

Electrical Engineering

School of Engineering

2-19-2007

\title{
Monte Carlo simulation of scanning electron microscopy bright contrast images of suspended carbon nanofibers
}

Makoto Suzuki

Toshishige Yamada

Santa Clara University, tyamada@scu.edu

Cary Y. Yang

SantaClara University, cyang@scu.edu

Follow this and additional works at: https://scholarcommons.scu.edu/elec

\section{Recommended Citation}

M. Suzuki, T. Yamada, and C.Y. Yang, "Monte Carlo Simulation of SEM Bright-contrast Images of Suspended Carbon Nanofibers," Applied Physics Letters 90, 083111(3 pp) (2007). https:// doi.org/10.1063/1.2450655

Copyright $@ 2007$ American Institute of Physics Publishing. Reprinted with permission.

This Article is brought to you for free and open access by the School of Engineering at Scholar Commons. It has been accepted for inclusion in Electrical Engineering by an authorized administrator of Scholar Commons. For more information, please contact rscroggin@scu.edu. 


\title{
Monte Carlo simulation of scanning electron microscopy bright contrast images of suspended carbon nanofibers
}

\author{
Makoto Suzuki, a) Toshishige Yamada, , ${ }^{\text {b) }}$ and Cary Y. Yang \\ Center for Nanostructures, Santa Clara University, 500 El Camino Real, Santa Clara, California 95053
}

(Received 22 November 2006; accepted 6 January 2007; published online 22 February 2007)

\begin{abstract}
The authors present a Monte Carlo study of previously observed bright contrast from carbon nanofibers suspended over the underlying substrate using scanning electron microscopy (SEM). The analysis shows that the origin of the bright contrast is mainly the increase in the secondary electron signal from the substrate when a gap between the nanofiber and substrate exists. The SEM signal dependence on the gap height is well reproduced by Monte Carlo simulation as well as a derived analytical expression. The bright contrast prevails when the SEM beam size is much smaller than the nanofiber diameter. (C) 2007 American Institute of Physics. [DOI: 10.1063/1.2450655]
\end{abstract}

Scanning electron microscopy (SEM) is a powerful tool not only for surface observation but also for analysis of the subsurface structure of materials. ${ }^{1-4}$ A recent experiment ${ }^{4}$ has shown that the SEM contrast formed by the secondary electrons (SEs) from carbon nanofibers (CNFs) prepared on the Si substrate is sensitive to their relative configuration. A typical bright contrast image and the corresponding side view are shown in Figs. 1(a) and 1(b), exhibiting a bright contrast at the CNF portion where it is not in contact with the underlying substrate. In Ref. 4, it is suggested that the different contrast due to the different configuration would be related to the electron range ${ }^{5} R(E)$ of beam energy $E$. According to this view, when $R(E)$ is larger than the CNF diameter $d$, the primary electron (PE) beam penetrates into the CNF and SEs are produced deep inside the underlying substrate, as shown in Fig. 1(c). The SEs in the substrate are emitted only when there is a finite gap with the height of $H$ between the CNF and substrate, resulting in the observed bright contrast [Figs. 1(a) and 1(b)]. However, there have been no models for Ref. 4 and how the contrast changes for different $H$ 's under which condition has not yet been studied systematically.

In this letter, we will discuss the role of $H$ on the contrast. As a starting point, we first derive a simple formula by assuming that the detector captures SEs only from the direction $\phi$ not covered by the CNF, as indicated in Fig. 1(c) and considering whether the SE signal from the substrate is blocked (dark contrast) or unblocked (bright contrast) in the presence of CNF. Using the parameters in Fig. 1(c) and from the emission angle $\theta_{E}$ defining the SE's escape window $\theta_{E}=\arccos [d /(2 H+d)]$ and $r=d / 2$, the SE signal increase $\Delta N$ corresponding to the contrast change is estimated with the cosine distribution ${ }^{6}$ as

$$
\Delta N \propto 2 \int_{\pi / 2-\theta_{E}}^{\pi / 2} \cos \phi \mathrm{d} \phi=\frac{4 H}{2 H+d}=\frac{2 H}{H+r} .
$$

Equation (1) shows that the SE signal is weak for small $H$ and increases and saturates for larger $H$. This intuitive discussion already catches the essence of the experiments but needs further investigation to address the assumption that the SE signal increase comes only from the substrate. In prac-

\footnotetext{
${ }^{\text {a) }}$ Author to whom correspondence should be addressed; electronic mail: m1suzuki@scu.edu

b) Also at NASA Ames Research Center, Moffett Field, CA 94035.
}

tice, there could be a different contribution than the substrate, e.g., the tertiary electrons which are to be generated by electrons reentering the CNF from the substrate. ${ }^{4}$ Moreover, the beam spot size $\left(D_{p}\right)$ dependence on the SEM contrast should also be clarified, since the voltage contrast ${ }^{7}$ and the electron-beam-induced conductivity ${ }^{8}$ have been reported as relevant contrast mechanisms in single-walled carbon nanotubes (SWCNTs), where $d$ is comparable with or smaller than $D_{p}$. Thus the relation between the present and existing models ${ }^{1,8}$ needs further investigation.

We present a systematic Monte Carlo study to elucidate the points above. We will demonstrate that our intuitive discussion in Eq. (1) is in fact valid as a first-order approximation and show that the SE signal increase indeed comes mainly from the substrate under CNFs, where the $H$ dependence is consistently explained by the increase in the SE emission angle. We will also demonstrate that $D_{p} \ll d$ is necessary in order to see bright contrast through the mechanism discussed in this letter.

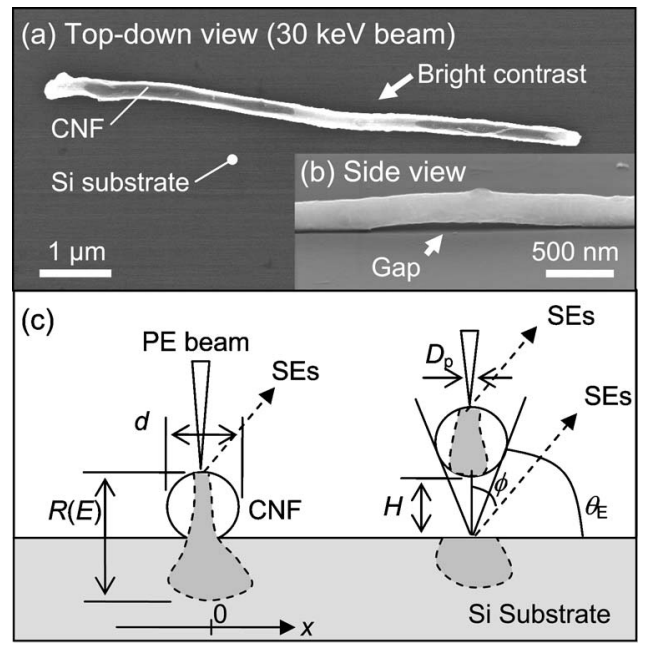

FIG. 1. (a) Top-down SEM image of a CNF on substrate, with beam energy of $30 \mathrm{keV}$. The arrow indicates the bright contrast portion. (b) Side view of the same CNF, exhibiting the clear gap at the bright contrast portion in (a). (c) Mechanism for bright contrast in CNFs on substrate. The PE beam is scanned perpendicular to the CNF axis ( $x$ direction). The CNF diameter is $d$, the electron range is $R(E)$, the gap height is $H$, and the beam spot size is $D_{p}$. For a finite $H$ (right panel), the SE emission angle $\theta_{E}$ is finite, resulting in the SE signal increase. 
Our Monte Carlo model gathers statistics on the trajectories of PEs suffering elastic scatterings during their gradual energy-losing flight because of inelastic processes. The scattering is described by the Mott cross-section formulation ${ }^{9}$ with the stochastic scattering angle and rate generated with random numbers, and the flight by the continuously slowingdown approximation ${ }^{10}$ based on Bethe's work ${ }^{11}$ describes the PE energy loss per unit flight distance, $|\mathrm{d} E / \mathrm{d} l|$. The PEs are traced until their energy becomes lower than $20 \mathrm{eV}$ or until they escape from the specimen as backscattered electrons. We assume that all energy loss of PEs during each Monte Carlo step length $\Delta l$ is converted to the energy for the SE generation, and the number of SEs emitted from the surface, $n_{\mathrm{SE}}$, decays exponentially with a mean free path $\lambda_{\mathrm{SE}}$ from the original value $n_{\mathrm{SE}}^{0}$ at the generation point, when SEs travel the distance $s$ to the surface. Thus the $n_{\mathrm{SE}}$ is obtained as follows: ${ }^{6}$

$$
n_{\mathrm{SE}}=n_{\mathrm{SE}}^{0} \exp \left(-s / \lambda_{\mathrm{SE}}\right), \quad n_{\mathrm{SE}}^{0}=\left|\frac{\mathrm{d} E}{\mathrm{~d} l}\right| \frac{\Delta l}{\varepsilon},
$$

where $\varepsilon$ is a mean generation energy for a single SE. The values of $\varepsilon$ and $\lambda_{\mathrm{SE}}$ are fitted to reproduce the experimentally observed SE emission coefficients ${ }^{12}$ of bulk graphite and $\mathrm{Si}$, yielding $\varepsilon=120 \mathrm{eV}$ and $\lambda_{\mathrm{SE}}=5 \mathrm{~nm}$ for $\mathrm{CNF}$, and $\varepsilon=65 \mathrm{eV}$ and $\lambda_{\mathrm{SE}}=2.5 \mathrm{~nm}$ for $\mathrm{Si}$ substrate. These values are consistent with those evaluated in Ref. 13 based on similar assumptions. The angular distribution of SEs emitted from the surface is assumed to obey the cosine distribution, ${ }^{6}$ $n_{\mathrm{SE}} \approx \cos (\phi)$, where $\phi$ is defined as the transverse angle of the SE emission measured from the surface normal. We consider the number of captured SEs by the detector as the SEM signal. Since a through-the-lens SE detector is used in the experiment, ${ }^{4}$ we assume that the emitted SEs are captured by the detector without any angular preference except those colliding to the sample again. $D_{p}$ is defined as the full width at half maximum of a Gaussian beam profile and is fixed at $1.0 \mathrm{~nm}$ unless otherwise stated. The specimen charging can be an issue when CNFs or CNTs are on the insulating substrate $^{7}$ and in the case of strong charging with a large electric field inside the specimen ${ }^{14}(>100 \mathrm{~V} / \mu \mathrm{m})$, PE trajectories are indeed affected by the field. Our experiment, ${ }^{4}$ however, was performed for the conductive nanofibers on the conductive substrate, and the effect of charging is not observed in the SEM contrast. Thus the resulting potential due to the charge builtup is negligible $(\ll 1 \mathrm{~V}$ at $100 \mathrm{~nm}$ feature size) and here we do not consider the local electric fields inside and outside the sample.

Figures 2(a) and 2(b) show the $50 \mathrm{PE}$ trajectories in the CNF $(d=100 \mathrm{~nm})$ with the gap heights of $H=0$ and $100 \mathrm{~nm}$, respectively. The trajectories with the beam energy of $1 \mathrm{keV}$ are plotted in black lines and those with $30 \mathrm{keV}$ beam in gray lines. While the PEs are forced to stop inside the CNF immediately at $1 \mathrm{keV}[R(E)<d]$, they penetrate it and are injected into the substrate with a small spatial spread at $30 \mathrm{keV}[R(E) \sim 50 d]$. Thus at $30 \mathrm{keV}$, the PE paths at the substrate surface are exposed to the vacuum with increasing $H$, as shown in Fig. 2(b). In Figs. 2(c) and 2(d), the computed SE signal profiles with and without the gap are shown. Each data point is obtained by 5000 incident PEs. At $1 \mathrm{keV}$ [Fig. 2(c)], the SE signal is independent of the gap height as expected. At $30 \mathrm{keV}$ [Fig. 2(d)], the SE signal increases

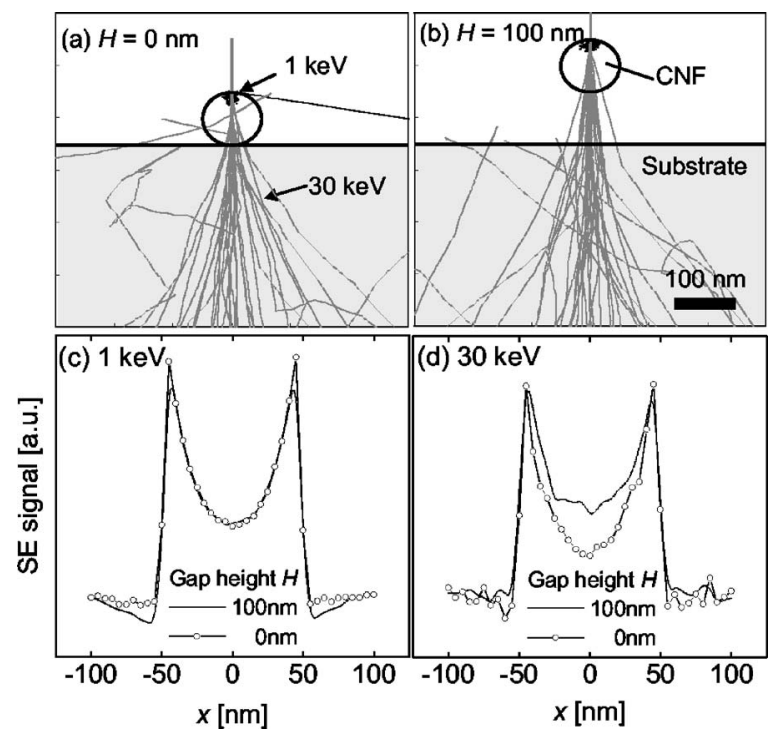

FIG. 2. $50 \mathrm{PE}$ trajectories with beam energies of $1 \mathrm{keV}$ (black lines) and $30 \mathrm{keV}$ (gray lines) and gap heights of (a) $H=0$ and (b) $100 \mathrm{~nm}$. SE signal profiles for beam energies of (c) $1 \mathrm{keV}$ and (d) $30 \mathrm{keV}$. The curve with open circles is for $H=0 \mathrm{~nm}$ and the solid curve for $100 \mathrm{~nm}$, respectively. The CNF diameter is $100 \mathrm{~nm}$. $5000 \mathrm{PE}$ trajectories are computed to obtain each data point.

with the gap height, reproducing the experimental results in Ref. 4.

To study the origin of the bright contrast, the SE emission distribution for the stationary beam at $x=0$ (CNF center) is computed separately from the $\mathrm{CNF}$ and the substrate. Figure 3 shows the number of SEs emitted from the CNF and the substrate for 10000 incident PEs. While $R(E)>d$ is valid for both energies, the SE signal from the CNF is not affected by the value of $H$ [Figs. 3(a) and 3(b)]. On the other hand, Figs. 3(c) and 3(d) show that the SE signal from the substrate at small $x$ increases sharply with $H$, showing that the bright image contrast comes mainly from the $\mathrm{Si}$ substrate. The other possible signals speculated in Ref. 4 including the tertiary electrons are found to be negligible $(<1 \%)$. The ratio of the $\mathrm{SE}$ signals from the CNF to those from the substrate is larger at $3 \mathrm{keV}$ than that at $30 \mathrm{keV}$. This is caused by the lower energy beam having larger elastic scattering cross section, ${ }^{9}$ resulting in larger beam spread in the CNF. Thus the weak-

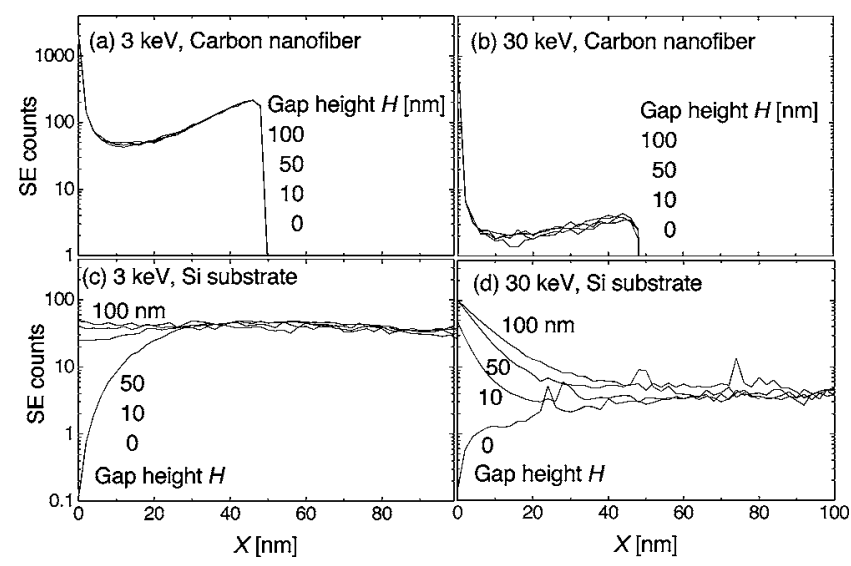

FIG. 3. SE emission distribution from CNF for beam energies of (a) $3 \mathrm{keV}$, and (b) $30 \mathrm{keV}$ and that from Si substrate for (c) $3 \mathrm{keV}$ and (d) $30 \mathrm{keV}$. 10000 PEs are injected at $x=0$, and the CNF diameter is $100 \mathrm{~nm}$. 

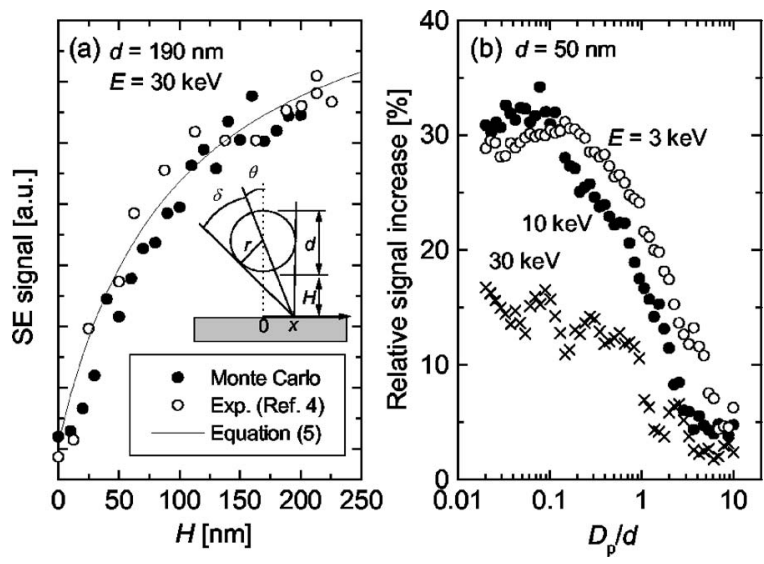

FIG. 4. (a) Gap height dependence of SE signal with $d=190 \mathrm{~nm}$ and $E=30 \mathrm{keV}$. Closed circles are Monte Carlo data, open circles are experimental data (Refs. 14 and 15), and the solid curve is an analytical fit using Eq. (5). Inset defines variables in Eqs. (3)-(5). (b) Beam size dependence of relative SE signal increase for $\mathrm{CNF}$ of $50 \mathrm{~nm}$ diameter. Open circles are for $E=3 \mathrm{keV}$, closed circles for $10 \mathrm{keV}$, and crosses for $30 \mathrm{keV}$. $5000 \mathrm{PE}$ trajectories are computed to obtain each Monte Carlo data point in (a) and (b).

ened bright contrast was observed ${ }^{4}$ at low $E$ even when $R(E)>d$.

The $H$ dependence of the SE signal for the beam at $x=0$ is computed and plotted in Fig. 4(a) with closed circles as well as experimentally observed values ${ }^{15}$ (open circles). $d$ is $190 \mathrm{~nm}$ for both data. Each Monte Carlo data point is obtained by computing 5000 incident PE trajectories. The sharp increase is well reproduced, showing that this contrast mechanism is indeed sensitive to the existence of a tiny gap. Now we can generalize Eq. (1) by taking into account the beam spread caused by the CNF. The number of SEs emitted from the substrate at $x, N_{\text {sub }}(x)$, is obtained in the same way as in Eq. (1):

$$
\begin{aligned}
N_{\mathrm{sub}}(x) & \propto \int_{\theta+\delta}^{\pi / 2} \cos \phi \mathrm{d} \phi+\int_{-\pi / 2}^{\theta-\delta} \cos \phi \mathrm{d} \phi \\
& =2\left[1-\frac{r(r+H)}{x^{2}+(r+H)^{2}}\right],
\end{aligned}
$$

where the angles $\theta$ and $\delta$ are defined in the inset of Fig. 4(a). If we assume that the surface SE emission from the substrate decreases with $x$ exponentially with a characteristic length $\lambda$, the total SE signal increase $\Delta N$ becomes

$$
\begin{aligned}
\Delta N & \propto \int_{0}^{\infty}\left[1-\frac{r(r+H)}{x^{2}+(r+H)^{2}}\right] \exp \left(-\frac{x}{\lambda}\right) \mathrm{d} x \\
& =\lambda-r[\sin (u) \operatorname{Ci}(u)-\cos (u) \operatorname{si}(u)],
\end{aligned}
$$

where the trigonometric integrals, $\operatorname{Ci}(u)=-\int_{u}^{\infty}(\cos t) / t \mathrm{~d} t$ and $\operatorname{si}(u)=-\int_{u}^{\infty}(\sin t) / t \mathrm{~d} t$, are used and $u=(r+H) / \lambda$. Taking the limit of $u \gg 1(\lambda \ll r)$ valid for $E=30 \mathrm{keV}$, we arrive at ${ }^{16}$

$$
\begin{aligned}
\Delta N & \propto \lambda-r \frac{1}{u}\left(1-\frac{2}{u^{2}}\right) \\
& =\lambda\left(\frac{H}{r+H}\right)\left[1+\frac{2 r}{H}\left(\frac{\lambda}{r+H}\right)^{2}\right] \quad(u \gg 1) .
\end{aligned}
$$

The first term is simply Eq. (1) and the second term is a correction to it. The prediction given by Eq. (5) (solid curve) generalizing the intuitive model of Eq. (1) compares well with experimental and Monte Carlo results as shown in Fig. 4(a).

Finally, we discuss the influence of the ratio $D_{p} / d$ on the relative signal difference, defined as the difference between the signal for the finite gap height (here, we set $H=d$ ) and that for $H=0$, divided by the average intensity. In Fig. 4(b), the relative signal difference is plotted against $D_{p} / d .5000$ $\mathrm{PE}$ trajectories are used to obtain each data point. We restrict the values of $d$ to be larger than the PE mean free path of $1-10 \mathrm{~nm}$ to satisfy the requirements of our model and fix $d=50 \mathrm{~nm}$ while changing $D_{p}$ instead. At any values of $E$, the signal difference drops at around $D_{p} \sim d$; this means that the nonlocal SE generation from the substrate far from the CNF dominates over the local SE increase under the CNF. This indicates that the SWCNTs with small diameters comparable with SEM beam size $(1-5 \mathrm{~nm})$ do not exhibit the bright image contrast through the present mechanism but possibly through other mechanisms. ${ }^{7,8}$

In summary, we have performed Monte Carlo simulations and studied the $\mathrm{SE}$ signal profile from $\mathrm{CNF}$ on a $\mathrm{Si}$ substrate. The bright SE signal mainly comes from the substrate and the signal is enhanced only in the presence of a finite gap between the CNF and substrate. The gap height dependence of the contrast is well reproduced by the Monte Carlo results as well as the analytical expression derived from considering the increase in the SE emission angle. The contrast mechanism prevails only when the SEM beam spot size is much smaller than the nanofiber diameter. For application to other nanowires such as small diameter nanotubes based on the present mechanism, the SEM beam spot must be reduced appreciably compared to the nanotube diameter.

The authors are grateful to Yusuke Ominami and Quoc Ngo for fruitful discussions.

\footnotetext{
${ }^{1}$ M. Nagase, A. Fujiwara, K. Kurihara, and H. Namatsu, Jpn. J. Appl. Phys., Part 1 42, 318 (2003).

${ }^{2}$ L. M. Gignac, M. Kawasaki, S. H. Boettcher, and O. C. Wells, J. Appl. Phys. 97, 114506 (2005).

${ }^{3}$ S. Jesse, M. A. Guillorn, I. N. Ivanov, A. A. Puretzky, J. Y. Howe, P. F. Britt, and D. B. Geohegan, Appl. Phys. Lett. 89, 013114 (2006).

${ }^{4}$ M. Suzuki, Y. Ominami, Q. Ngo, C. Y. Yang, T. Yamada, A. M. Cassell, and J. Li, J. Appl. Phys. 100, 104305 (2006).

${ }^{5}$ K. Kanaya and S. Okayama, J. Phys. D 5, 43 (1972).

${ }^{6}$ H. Seiler, J. Appl. Phys. 54, R1 (1983).

${ }^{7}$ T. Brintlinger, Y. F. Chen, T. Dürkop, E. Cobas, M. S. Fuhrer, J. D. Barry, and J. Melngailis, Appl. Phys. Lett. 81, 2454 (2002).

${ }^{8}$ Y. Homma, S. Suzuki, Y. Kobayashi, M. Nagase, and D. Takagi, Appl. Phys. Lett. 84, 1750 (2004).

${ }^{9}$ Z. Czyżewski, D. O. MacCalium, A. Roming, and D. C. Joy, J. Appl. Phys. 68, 3066 (1990).

${ }^{10}$ D. C. Joy and S. Luo, Scanning 11, 176 (1989).

${ }^{11}$ H. Bethe, Ann. Phys. 5, 325 (1930).

${ }^{12}$ The set of the experimental data of the SE emission coefficients is available at http://web.utk.edu/ sructk/htm/interact.htm.

${ }^{13}$ D. C. Joy, Monte Carlo Modeling for Electron Microscopy and Microanalysis (Oxford, New York, 1995), Chap. 8, p. 164.

${ }^{14}$ M. Kotera and H. Suga, J. Appl. Phys. 63, 261 (1988).

${ }^{15}$ The data are extracted from the original images of Fig. 2 in Ref. 4.

${ }^{16}$ M. Abramowitz and I. A. Stegun, Handbook of Mathematical Functions with Formulas, Graphs, and Mathematical Tables (Dover, New York,
} 1965), pp. 231-233. 